\title{
Inestabilidad residual de tobillo en pacientes con lesión de la sindesmosis sin fractura tratados con tornillos situacionales
}

\author{
Residual ankle instability in patients with syndesmosis lesions \\ without fracture treated with situational screws
}

\author{
Castro-Guerrero DE,* Rosas-Medina JA ${ }^{\ddagger}$
}

Hospital General Regional Núm. 2, Instituto Mexicano del Seguro Social, Ciudad de México.

\begin{abstract}
RESUMEN. Introducción: La lesión de la sindesmosis tibioperonea se presenta por lo regular acompañada de la fractura de los maléolos ya sea medial o lateral, muy rara vez la sindesmosis puede lesionarse sin que exista una fractura de alguna de las estructuras óseas que conforman el tobillo, representa alrededor de $1 \%$ de todas las lesiones. Al ser muy raras, no se diagnostican en el evento agudo y suelen tratarse como un simple esguince de tobillo. Material y métodos: Serie de casos con lesión de la sindesmosis sin fractura de tobillo, tratadas con colocación de doble tornillo situacional, apoyo diferido y retiro de los implantes a los dos meses. Posteriormente, a los seis meses de la cirugía inicial se aplica el test de inestabilidad de tobillo de Cumberland (CAIT), el cual cuenta con nueve reactivos donde se mide el grado de inestabilidad del tobillo. Resultados: Durante un año se detectaron cuatro casos de lesión de la sindesmosis sin fractura de un total de 349 casos quirúrgicos tratados en el hospital, exclusivamente en pacientes masculinos, todos ellos menores de 40 años. Seis meses después de la cirugía se aplicó el CAIT encontrando una inestabilidad residual en $100 \%$ de los casos tratados, algunos en mayor medida que los demás. Discusión: Este resultado es poco alentador y nos hace reconsiderar el tratamiento establecido para mejorar la estabilidad final del tobillo.
\end{abstract}

Palabras clave: Tobillo, sindesmosis, tratamiento, lesión, tornillo.

\section{Nivel de evidencia: IV}

${ }^{*}$ Residente de $4^{\circ}$ año de Ortopedia.

¥ Jefe de Servicio de Miembro Pélvico.

Hospital General Regional Núm. 2, Instituto Mexicano del Seguro Social.

Dirección para correspondencia:

Dr. David Emmanuel Castro Guerrero

Calzada de las Bombas 121, Departamento 301,

Ex-Hacienda Coapa, C.P. 04890, Alcaldía Coyoacán, Ciudad de México. Tel: 833-2160101

E-mail: dcastrog@gmail.com

Este artículo puede ser consultado en versión completa en: www.medigraphic.com/actaortopedica
ABSTRACT. Introduction: The lesion of the distal tibiofibular syndesmosis is commonly accompanied by the fracture of the maleollus either medial or lateral, rarely, the syndesmosis can be injured without there being a fracture of any of the bone structures that make up the ankle, accounting for about $1 \%$ of all injuries. Being very rare, they are not diagnosed at the acute event, and are usually treated as a simple sprained ankle. Material and methods: Series of cases with ankle fractureless syndesmosis lesion, treated with situational double screw placement, deferred support and implant removal at two months. After six months of initial surgery, the Cumberland ankle instability (CAIT) test is applied which measures the degree of ankle instability. Results: For one year, 4 cases of fractureless synosmosis lesions were found out of a total of 349 surgical cases treated in the hospital, exclusively in male patients, all under the age of 40 . Six months after surgery, CAIT was applied, encountering residual instability in $100 \%$ of treated cases. Discussion: This result is unencouraging and makes us reconsider the treatment established to improve the final stability of the ankle.

Keywords: Ankle, syndesmosis, treatment, injury, screw.

\section{Introducción}

La articulación del tobillo está formada por la parte distal de la tibia, peroné y domo del astrágalo. La tibia y el peroné forman una mortaja donde encaja el astrágalo y que, junto a la cápsula, conforman la articulación del tobillo propiamente dicha y permite la movilidad de la articulación. Existe una articulación sin cartílago entre la tibia y peroné que está formada sólo por tejido fibroso que une estos dos huesos en sus caras lateral y medial respectivamente, esta porción anatómica del tobillo es la sindesmosis, la cual está formada por un ligamento tibioperoneo anterior, membrana interósea, ligamento tibioperoneo inferior y ligamento transverso inferior, 
el cual es una porción distal del ligamento tibioperoneo anterior. ${ }^{1}$ Los demás ligamentos del tobillo se conforman por un grupo medial llamado en conjunto ligamento deltoideo, con un fascículo superficial con fibras que van desde la tibia hasta su inserción distal en el escafoides tarsal, sustentaculum tali y la porción posterior del astrágalo. Las fibras profundas conforman el ligamento tibioastragalino profundo. En el grupo lateral, las fibras ligamentosas van desde el maléolo peroneo y conforman los ligamentos peroneoastragalino anterior, calcaneoperoneo y peroneoastragalino posterior ${ }^{2}$ (Figura 1). La unión de estos ligamentos conforman un anillo fibroso que mantiene la congruencia articular del tobillo y es

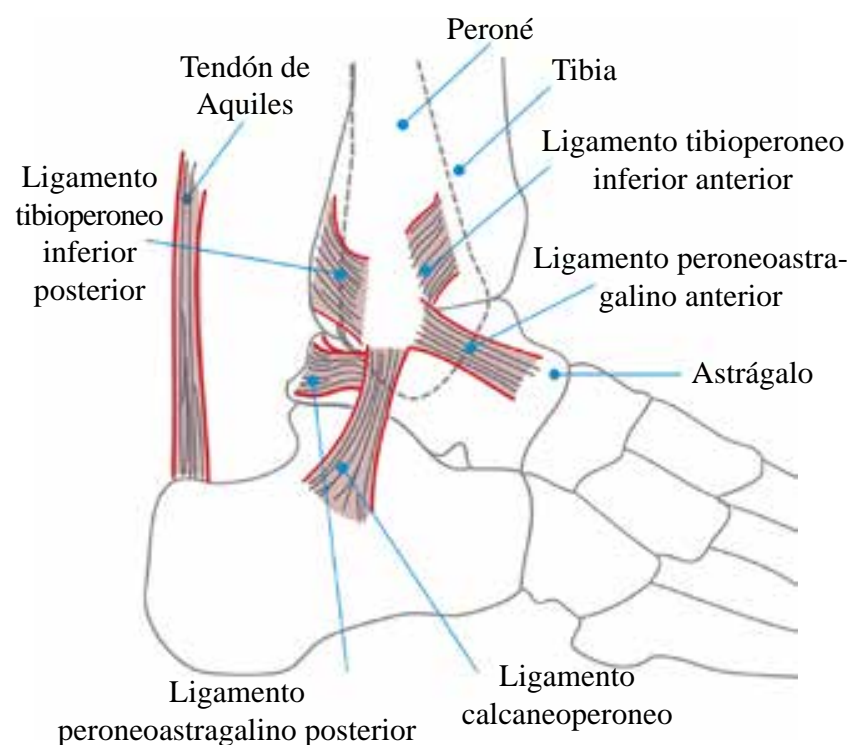

Figura 1: Complejo ligamentario del tobillo, vista lateral. la responsable de permitir que el pie se adapte a los diferentes terrenos que pisa. Una incongruencia articular secundaria a fracturas o a una inestabilidad ligamentaria por esguinces pueden tener como consecuencia una mala recuperación y una artrosis temprana.

Los ligamentos del tobillo otorgan la estabilidad a la articulación, sobre todo cuando el pie se encuentra en flexión plantar, ya que en flexión dorsal, la forma del astrágalo permite mayor congruencia articular en la mortaja y por lo tanto, mayor estabilidad. Los ligamentos del complejo medial evitan el excesivo prono y los del complejo lateral limitan el supino. ${ }^{3,4}$ Cuando existe un mecanismo lesional de supino o prono, los ligamentos que limitan dicha función pueden lesionarse o en algunos casos puede haber fractura del maléolo comprometido. Estas fuerzas lesionales pueden acompañarse de aducción, rotación externa y abducción y conformar varias lesiones complejas en el tobillo, las cuales están descritas por la clasificación de Lauge-Hansen.

En cuanto a la sindesmosis, la función estabilizadora está conferida en 35\% por ligamento tibioperoneo anterior, siendo el ligamento que se lesiona con más frecuencia. El ligamento tibioperoneo posterior otorga hasta $60 \%$ de estabilidad y muy rara vez se lesiona. El restante $5 \%$ de la estabilidad la aporta la membrana interósea. ${ }^{5}$ La sindesmosis se puede lesionar muy comúnmente junto a las fracturas de los maléolos y cuando no se observe una fractura en los maléolos, pero exista una diástasis de la sindesmosis, debe sospecharse una fractura del peroné proximal (Maisonneuve), la cual tiene un mal pronóstico, ya que implica una lesión masiva de la sindesmosis y un compromiso de la estabilidad del tobillo. Debido a que el ligamento deltoideo es el principal estabilizador del tobillo, una lesión de la sindesmosis acompañada de lesión del ligamento deltoideo

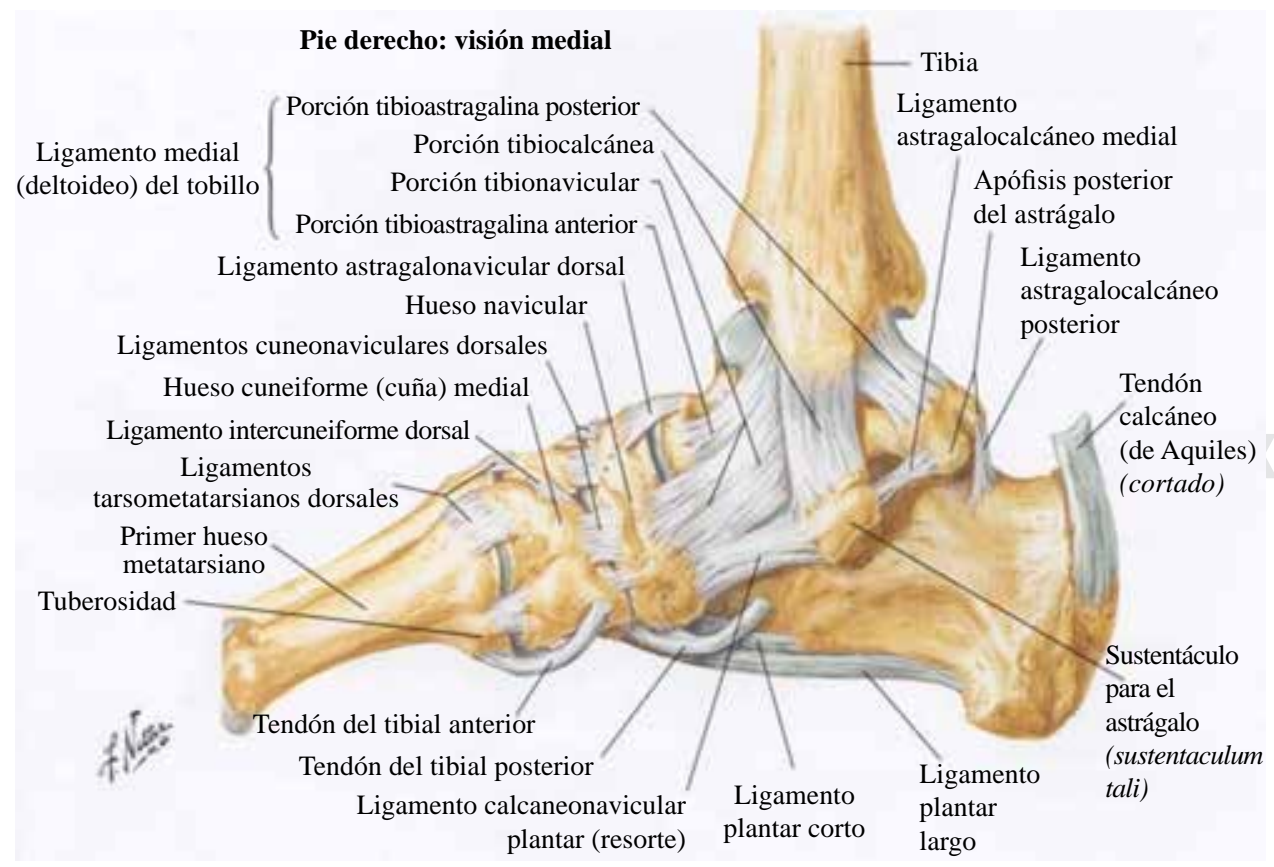

Figura 2:

Complejo medial del tobillo y ligamento deltoideo. 

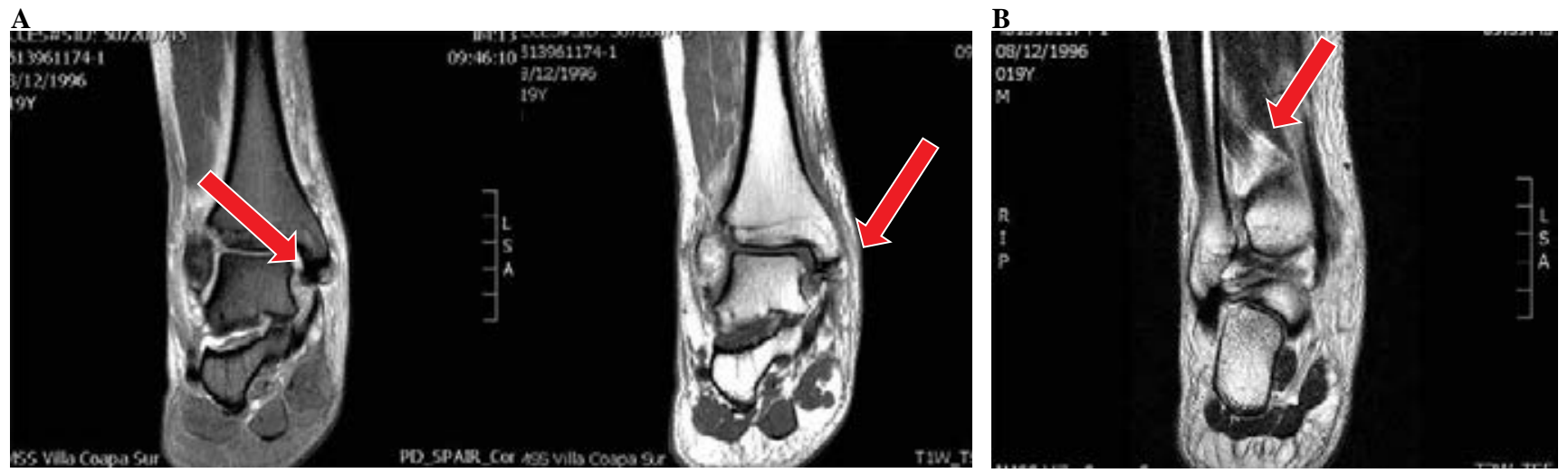

Figura 3: A) Se observa IRM con lesión del complejo medial, ligamento deltoideo (flecha). B) Se observa una señal hiperintensa a nivel de la sindesmosis (flecha).

puede comprometer aún más la estabilidad y la congruencia de la mortaja. En un estudio cadavérico se encontró que la lesión completa y única de la sindesmosis en el tobillo de un cadáver produce una diástasis de la mortaja de $2 \mathrm{~mm}$ y que cuando esta lesión se acompaña de la sección del complejo deltoideo profundo, la diástasis puede ampliarse hasta 3.7 $\mathrm{mm}$, esto corrobora la importancia del ligamento deltoideo (Figura 2). ${ }^{6}$

La lesión de la sindesmosis y del ligamento deltoideo o complejo lateral sin fractura de los maléolos o del peroné proximal es muy rara (Figura 3). En la literatura se reporta que la lesión del complejo ligamentario del tobillo sin fractura tiene una incidencia de $1 \%$ de todas las lesiones del tobillo. Las lesiones ligamentarias sin fracturas tienden a pasar desapercibidas y tienen un mal pronóstico funcional, pues el tobillo pierde estabilidad y se predispone a una artrosis temprana, dolor residual y pérdida de la fuerza. La razón es que las lesiones sin fractura requieren un alto grado de sospecha de acuerdo al mecanismo de lesión (rotación externa del pie sobre el tobillo). Edwards clasifica las lesiones de tobillo sin fractura en cuatro tipos: la tipo I son lesiones acompañadas de subluxación lateral del peroné sin deformidad plástica de la misma que pueden ser tratadas con una reducción abierta, retiro de tejido interpuesto y colocación de tornillo situacional. La tipo II se presenta con una subluxación lateral del peroné acompañada de una deformidad plástica que requiere osteotomía previa a una reducción abierta y fijación interna con tornillo situacional. La tipo III es poco común y se debe a una subluxación posterior con rotación del peroné. En la tipo IV el astrágalo sufre una luxación superior, lo que resulta en una divergencia tibioperonea con lesión de la sindesmosis. La tipo III y tipo IV pueden tratarse con reducción cerrada y colocación de aparato de yeso. La tipo I y tipo II requieren un tratamiento quirúrgico.?

Algunas lesiones son francas y pueden ser demostradas con la radiografía simple, otras requieren de una radiografía con estrés para hacerla visible. En este caso, una prueba con rotación externa del pie puede hacer visible una lesión sindesmal que pasaría desapercibida en la radiografía sim- ple. Nosotros recomendamos la radiografía simple y comparativa con el tobillo sano, ya que una prueba con estrés puede ser demasiado dolorosa para que el paciente la tolere, aunque en algunos casos puede ser necesaria para descartar la lesión, por lo que dadas las circunstancias, sugerimos una prueba con estrés a tolerancia, pidiéndole al paciente que apoye su tobillo afectado evitando el dolor intenso. Otra maniobra diagnóstica incluye el cajón que demuestra inestabilidad del tobillo, la prueba de la rotación externa que consiste en estabilizar la rodilla en $90^{\circ}$ y realizar una movilidad pasiva con rotación externa del pie, la prueba es positiva cuando se produce dolor a nivel de la sindesmosis. ${ }^{8} \mathrm{La}$ prueba de compresión (squeeze test) de Hopkins consiste en efectuar compresión en un punto por encima de la parte media de la pierna a nivel del espacio tibioperoneo, esto ocasiona separación del peroné además de dolor exquisito en la zona afectada. ${ }^{8,9}$ Se puede utilizar una resonancia magnética aumentando la especificidad de la lesión.

Las lesiones sindesmales pueden acompañarse de otras lesiones, tales como fracturas de alguno de los maléolos y de lesiones ligamentarias del complejo lateral o medial. Hay que recordar que como ligamentos pueden sufrir esguinces de diferentes grados de complejidad. Por lo tanto, cuando hay una lesión de tobillo sin fractura, es sinónimo de una estela de esguinces de diferentes grados que puede afectar a diferentes ligamentos en sus distintas profundidades, por consiguiente puede existir una innumerable cantidad de lesiones diferentes de acuerdo a los ligamentos involucrados, la intensidad del esguince y si existe o no fractura de algún maléolo. Por tanto, referirse a «esguince de tobillo» puede resultar en un término que no aporta mayor información más que la lesión ligamentaria en una zona anatómica no específica. En la literatura anglosajona se utiliza el término high ankle sprain para describir un esguince de la sindesmosis, contra el low ankle sprain cuando el esguince ocurre en el complejo medial o lateral. También existe el término ankle diastasis que describe una inestabilidad tibioperonea distal que implica una lesión completa (o esguince grado III) de la sindesmosis.

Los tratamientos propuestos son varios y así como DeLee y Edwards propusieron la colocación de tornillo sindes- 
mal en el grado I y II, ${ }^{7}$ también se han realizado otros protocolos con el uso de Tightrope que han obtenido resultados favorables. ${ }^{9}$ El tratamiento conservador con aparato de yeso y reducción cerrada no son recomendables, debido a que el tobillo debe obtener una reducción anatómica que le permita soportar una carga constante y conservar el movimiento del pie para adaptarse a los diferentes terrenos. Los tratamientos artroscópicos también pueden ser una alternativa menos invasiva y han tenido buenos resultados en algunos casos. ${ }^{10}$

\section{Material y métodos}

Se realizó un seguimiento de los casos quirúrgicos con lesión y/o fractura de tobillo del servicio de miembro pélvico durante el lapso de un año, se encontraron sólo cuatro casos con una lesión de la sindesmosis tibioperonea distal sin fractura, la cual fue demostrada con radiografía comparativa, exploración clínica y corroborada durante el transquirúrgico. El mecanismo de lesión en todos los casos correspondió a una rotación externa del pie sobre la pierna. El manejo inicial en todos los casos fue inmovilización, después se efectuó cierre quirúrgico de la sindesmosis con doble tornillo situacional de $3.5 \mathrm{~mm}$ (Figura 4) y se instruyó diferir el apoyo. Los implantes fueron retirados en un plazo de seis semanas y se inició la marcha en casa. Posterior a los seis meses se realizó un seguimiento a los casos y se aplicó el CAIT.

El test de inestabilidad del tobillo de Cumberland (Cumberland Ankle Inestability Tool) o CAIT está formado por nueve reactivos con un valor en cada uno. El resultado final puede ir de 0 a 30 puntos, los puntos bajos demuestran inestabilidad severa. Un resultado de 27 o menos indica una probable inestabilidad de la extremidad medida. ${ }^{11}$

\section{Resultados}

Se detectaron cuatro casos con lesión de la sindesmosis sin fractura, todos del sexo masculino y menos de 40 años.
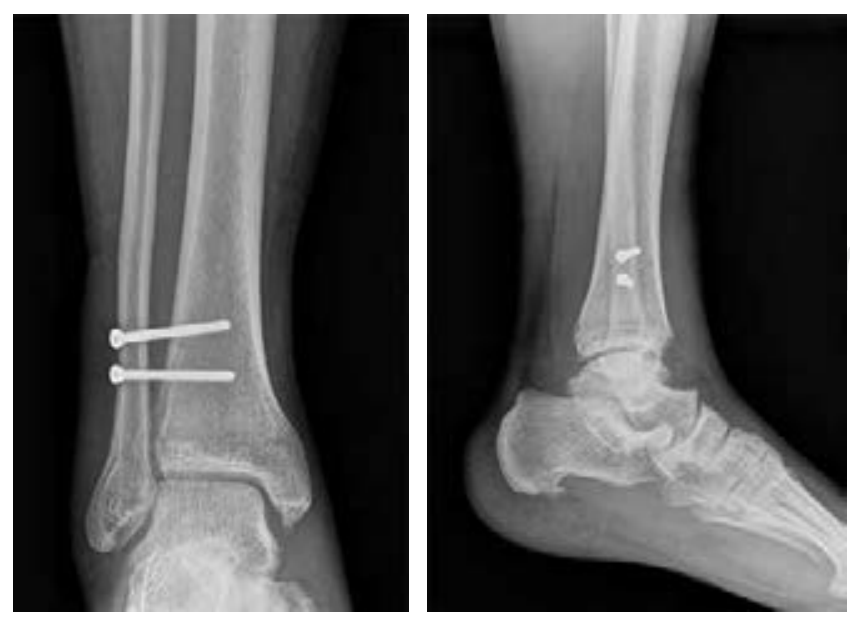

Figura 4: Resultados postquirúrgicos inmediatos.
En cuanto al resultado en el CAIT, 100\% de los casos obtuvo una calificación de 27 o menos, indicando una inestabilidad del tobillo. El resultado más bajo fue de 14 y el más alto de 25 puntos, siendo éste el resultado del paciente más joven (20 años). Dentro del CAIT se considera 30 puntos la máxima calificación y por lo menos 28 puntos o más como un indicativo de estabilidad normal en el tobillo.

De los cuatro pacientes, todos habían regresado a su actividad laboral/escolar. Tres de ellos se sentían satisfechos con la funcionalidad y sólo uno mostraba insatisfacción. En cuanto a la actividad deportiva, sólo tres pacientes habían reiniciado actividad física ligera y un paciente sentía que no podría regresar aún. No hubo complicaciones dentro de la serie de casos.

\section{Discusión}

El estudio demuestra que todos nuestros pacientes con lesiones aisladas de la sindesmosis tratados con dos tornillos situacionales van a resultar en inestabilidad articular, por lo menos a los seis meses después de su cirugía. El paciente más joven obtuvo la mejor calificación de estabilidad, por lo que la edad puede considerarse un factor de buen pronóstico. Dentro de las debilidades de este estudio está el corto tiempo de seguimiento (seis meses) y la falta de estudios de control, una terapia de rehabilitación no supervisada, ya que los pacientes fueron instruidos a reiniciar sus actividades normales una vez que se les retiraron los implantes. Es necesario realizar un estudio con una muestra más grande y con mayor tiempo de seguimiento, una técnica quirúrgica más depurada al igual que estudios más amplios de imagenología para el diagnóstico y el seguimiento así como una terapia de rehabilitación dedicada para obtener resultados satisfactorios.

Este estudio demuestra lo poco común de este tipo de lesiones, considerando su rareza y la similitud clínica con los esguinces de los complejos laterales o mediales. Es por esta razón que es necesario prevenir el subdiagnóstico de estas lesiones, ya sea con mejores estudios de imagen y/o con mejor seguimiento clínico, ya que los pacientes pueden recibir un tratamiento deficiente y acabar con inestabilidad residual de la articulación y una cirugía poco exitosa con las consecuencias que esto conlleva. Prueba de la escasa información acerca de esta lesión es la falta de un término adecuado. Podríamos darle el nombre de esguince alto de tobillo como en inglés high ankle sprain, lo que lo diferencia del esguince común de los complejos laterales, pero aun así es un término vago, pues no implica inestabilidad o lesión en algún ligamento en particular de los tantos que conforman el complejo ligamentario del tobillo. Es conveniente recalcar la rareza diagnóstica de esta lesión en nuestra institución así como la importancia de un diagnóstico oportuno. Debido a que puede confundirse con un esguince simple de tobillo, cualquier paciente en quien, por el mecanismo de lesión, se sospeche que existe una lesión de la sindesmosis sin fractura, deberá iniciarse un protocolo de estudio, descartando 
primero la fractura alta de peroné y posteriormente radiografías comparativas, radiografías con estrés a tolerancia, pasando por último por la resonancia magnética.

Es necesario iniciar otros estudios que nos ayuden a identificar las lesiones ligamentarias sencillas de aquéllas que producen inestabilidad del tobillo, para así darle tratamiento más completo a estas últimas y mejorar los resultados a largo plazo.

Bibliografía

1. Attarian DE, McCrackin HJ, DeVito DP, McElhaney JH, Garrett WE. Biomechanical characteristics of human ankle ligaments. Foot Ankle. 1985; 6: 54-8.

2. Leardini A, O’Connor JJ, Catani F, Giannini S. The role of passive structures in the mobility and stability of the human ankle joint: a literature review. Foot Ankle Int. 2000; 21(7): 602-15.

3. Bonnel F, Toullec E, Mabit C, Tourne Y, Sofcot. Chronic ankle instability: biomechanics and pathomechanics of ligaments injury and associated lesions. Orthop Tramatol Surg Res. 2010; 96(4): 424-32.
4. Brostrom L: Sprained ankles. Part 1: Anatomic lesions in recent sprains. Acta Chir Scand. 1964; 128: 483-95.

5. Boden SD, Labropoulos PA, McCowin P, Lestini WF, Hurwitz MSR. Mechanical considerations for the syndesmosis screw. A cadaver study. J Bone Joint Surg Am. 1989; 71: 1548-55.

6. Eyring EJ, Guthrie WD. A surgical approach to the problem of severe lateral instability at the ankle. Clin Orthop. 1986; 206: 185-91.

7. Edwards GS Jr., DeLee JC. Ankle diastasis without fracture. Foot Ankle. 1984; 4(6): 305-12.

8. Álvarez LA, Rodríguez RE, García LY, Muñoz IA. Lesiones de la sindesmosis tibioperonea distal: review article. Rev Cubana Ortop Traumatol. 2008; 22(1).

9. Willmott HJ, Singh B, David LA. Outcome and complications of treatment of ankle diastasis with tightrope fixation. Injury. 2009; 40(11): 1204-6.

10. Ogilvie-Harris DJ, Reed SC. Disruption of the ankle syndesmosis: diagnosis and treatment by arthroscopic surgery. Arthroscopy. 1994; 10(5): 561-8.

11. Hiller CE, Refshauge KM, Bundy AC, et al. The Cumberland ankle instability tool: a report of validity and reliability testing. Arch Phys Med Rehabil. 2006; 87: 1235-41. 